\title{
Incorporating negative and positive word of mouth (WOM) in compartment-based epidemiology models in a not-for-profit marketing context
}

\author{
John Andy Wood ${ }^{1}$ D
}

Revised: 8 February 2021 / Accepted: 16 May 2021 / Published online: 26 May 2021

(c) The Author(s), under exclusive licence to Springer Nature Limited 2021

\begin{abstract}
The simultaneous occurrence of negative and positive word of mouth is often likely in a marketing context. Measuring the influence of these conflicting social pressures is not straightforward in current diffusion models. Adaptations from compartment models of epidemiology can provide methods for estimating both positive and negative word of mouth. This study examines the impact of positive and negative word of mouth on donating behavior using data from over 89,000 households that made a gift to a non-profit. The 10-year longitudinal dataset creates the opportunity to calculate negative and positive word of mouth on donating behavior.
\end{abstract}

Keywords Word of mouth $\cdot$ Diffusion $\cdot$ Nonprofit $\cdot$ Longitudinal $\cdot$ Compartment models

COVID-19 has brought epidemiology and associated mathematical models into the public's consciousness. The pandemic heightened interest among marketers in diffusion models and their association with word of mouth. Many marketers likely have a passing familiarity with word of mouth models and their origins in models of contagious diseases. It may not be as common to realize that the extensiveness of the spread of word of mouth (WOM) communication flips the traditional epidemiology models of high transmission rates to a positive outcome in the marketing literature. These "informal communications directed at other consumers about the ownership, usage, or characteristics of particular goods and services and/or their sellers" (Westbrook 1987 p. 261) are desirable when they convey positive information.

The literature indicates that these messages have significant implications on first-time purchase behaviors in the marketplace and subsequently impact financial performance (Liu 2006; Luo 2007). While research on WOM exists in many other contexts, one stream in marketing has seen an

John Andy Wood

wood3ja@jmu.edu

1 College of Business, James Madison University, MSC 0205, Zane Showker Hall, Room 527, Harrisonburg, VA 22807, USA extensive focus on the influence of WOM. That research stream, along with its associated mathematical models, emphasizes the initial transaction of a new offering (Arndt 1967; de Matos and Rossi 2008), also known as the diffusion of innovation. These models of diffusion base their frameworks on mathematical models from epidemiology. Even the terminology for a successful digital WOM campaign, i.e., "going viral," reminds us of the link between WOM in diffusion models and the foundation of those same models in infectious disease models.

The current interest in modeling WOM is not strictly motivated by COVID-20. Recently, interest from marketing practitioners and researchers rose as the extensiveness of digital WOM became evident. The amplitude and magnitude of WOM through the use of e-platforms (Solis and Webber 2012), in particular, examines this effect. Additionally, research in the digital area suggests consumers view e-WOM as more credible and is seen as trustworthy (Liu 2006). These online findings build upon older literature that found the informal communications, which often occur in social networks (Brown and Reingen 1987), strongly influence sentiment. In marketing, it seems generally accepted that WOM has a powerful influence on consumer activities.

Additionally, at a conceptual level, the marketing literature clarifies that both positive and negative word of mouth are significant influences on market activities (Arndt 1967; Chevalier and Mayzlin 2006; Herr et al. 1991; Liu 
2006; Richins 1983). Researchers note that the interactions' valence can be both negative and positive (Liu 2006). Despite the frameworks suggesting both negative and positive word of mouth is relevant, a certain level of reliance by marketing diffusion models on the older generation of epidemiology mathematical models limits the literature. This reliance contributes to a gap in our understanding of the WOM phenomenon in marketing.

The focus on the positive influence of WOM likely develops from early mathematical models focusing on product diffusion and production adoption. The Bass Model of the diffusion of innovation sees WOM as a component of the social influence on 'imitators' to adopt a new product (Bass 1969). Imitators are those individuals adopting the product or service after early adopters. The discussion around these models typically does not include possible negative impacts from social pressure. However, recently, researchers suggest that solely relying on the Bass Model is insufficient in social networks. A solution introduced before and in this study is the introduce compartment-based epidemiology models. One such model is known as the $S I R$ (susceptibleinfectious-recovered) model. Previous studies suggest that researchers consider this model and the Bass diffusion model (Fibich 2016; Hethcote 2000) when accounting for social influence.

These base $S$ I $R$ models introduce population compartments to explain disease transmission (Feng et al., 2011). Recently, the COVID-19 pandemic, as well as other events such as the SARs epidemic of 2002-2003 (Brauer and Castillo-Chavez 2013), has seen the expanded interest and revisions of this $S I R$ model. These recent approaches in compartmental models allow for sub-categories in the populations such as susceptible, infectious, immunized, and recovered portions or compartments of the population. (Brauer and Castillo-Chavez 2013).

Looking at diffusion in the marketing context through the lens of $S I R$ models can be a step forward as these models can be adapted to allow for the simultaneous consideration of positive and negative word of mouth. In marketing, it seems that it has been difficult to measure both WOM types on a large scale. A small-scale empirical study that simultaneously incorporates positive and negative WOM in social networks exists (Stich et al., 2014). Despite this exception, researchers note that failing to estimate the model coefficients adequately or omitting negative WOM limits the generalizability of findings around positive and negative WOM in marketing (Mahajan et al. 1990, 1984; Tanny and Derzko 1988). The data in this paper represent over 89,000 individuals households and includes over one million discrete transactions. This paper develops empirical support for using compartment models to estimate and test negative word of mouth (NWOM). Using this large-scale sample with its empirical outcomes helps marketers consider the nature of the communication when positive, negative, or mixed WOM exists (de Matos and Rossi 2008).

We believe that simultaneously modeling positive and negative word of mouth in compartmental models such as $S$ $I R$ addresses a critical literature gap. This paper discusses the S I R model's assumptions and logic and provides an adaption applied to marketing. Longitudinal donation data from a non-profit organization provide empirical support that positive and negative WOM may occur within the same marketing context. This research paper examines both positive and negative WOM's implications in an adoption model in the marketing context.

We summarize these objectives as follows:

(a) Introduce, explicate, and adapt the $S I R$ model to marketing.

(b) Suggest a plausible framework explaining how changes in donor patterns indicate negative word of mouth in a non-profit context.

(c) Using a large-scale database of initial donating activity, examine empirical evidence of NWOM activity impeding donation activity.

\section{Background}

\section{Word of mouth in diffusion}

The concept that ideas and products spread or diffuse over time has a long history. Early models saw diffusion as approximately a normal curve (Pemberton 1936), which subsequently saw adoption in the marketing literature (Rogers 1962). Beyond proposing the shape of the diffusion trajectory, these models suggested that forces within society lead to the adoption of innovations. The original paper on modeling from Bass (1969) did not explicitly mention word of mouth; it did refer to the social pressures on the imitators influencing their adoption behavior. Throughout this stream of literature, some underlying assumptions about the composition and actions of subsets of the population during this diffusion process. These subsets seem analogous to the compartments of epidemiology compartment models.

Word of mouth is one social influence identified early in the literature as part of the new product adoption process (Arndt 1967). Since then, most diffusion models have word of mouth (WOM) as influencing not necessarily the first innovators but rather later adopters, the imitators, into trying the new product. In these models, the underlying inference is that the direction of the WOM influence is positive.

Negative word of mouth (NWOM) or communicating with others about displeasure with a product or provider is not new to the marketing literature. NWOM is often framed as one of several possible responses when the 
buyer is not satisfied with a marketing interaction (Richins 1983). These potential responses to dissatisfaction include choosing to no longer transact with the seller, engaging in complaining behavior, initiating NWOM, or accessing third parties such as lawyers or advocacy organizations (Charlett et al. 1995). A model with a limited sample size sought to add negative word of mouth as an influence (Mahajan, Muller, et al. 1984). Researchers suggest the reasons that individuals choose NWOM include but are not limited to the following:

(1) the desire to help others avoid a negative purchase experience (altruism);

(2) using the act of telling others about the negative experience as a coping mechanism for felt stress (anxiety relief);

(3) a need to share the experience with others to seek advice on alternatives courses of action (advice-seeking);

(4) a desire to limit the offending company's revenue stream (vengeance) (Edison and Geissler 2011; Sundaram et al. 1998; Wetzer et al. 2007b).

Additionally, word of mouth, whether positive or negative, has been seen as reinforcing social identity (Arndt 1967). Some research has linked NWOM to association goals through bonding and entertainment (Wetzer et al. 2007b). In the context of this research's data, the behavior of giving to charitable organizations likely leads to a glow from giving or guilt from an impulsive act (Taute and McQuitty 2004).

While dissatisfaction and remorse have an extensive literature base, capturing customers' or clients' expressions of this remorse can be challenging (Richins 1983). Research indicates that customers are much more likely to turn to Negative Word of Mouth rather than complain to the marketer (Chelminski and Coulter Robin 2011). Strictly relying on verbal expressions, discontent seems likely to limit the modeling of negative word of mouth.

From these various conceptualizations about the origins of negative word of mouth, it appears that the common trigger is a natural or perceived sense of not receiving the anticipated value from the purchase. This post-purchase dissatisfaction leading to negative word of mouth appears related to the literature on buyers' remorse perceptions. We suggest that contexts that have a clear delineation about value exchanged coupled with solid perceptions of social identity are good candidates to investigate the consequences of negative word of mouth.

\section{Negative word of mouth and donations}

One such context is donations to non-profit organizations. Charities and other non-profit organizations have been part of the marketing domain since Kotler and Levy's (1969) classic article, "Broadening the Concept of Marketing." These organizations have also recognized the importance of marketing to their fund-raising efforts and have adopted the marketing concept. For many of these charities and nonprofit organizations, their target audience is the individual donor, and that a successful value proposition must be present so the target audience can believe and financially support the cause. It is critical for the organization's marketing strategy to understand what the donor values and why they give to encourage long-term commitment (Guy and Patton 1989). Several studies have investigated these motivations to donate.

In non-profit marketing, WOM has a significant role in reinforcing social identity through behaviors such as donations (Arnett et al. 2003). Through informal conversations about the gift, communication confirms the speaker's membership in their targeted social identity group. Research indicates the perceived benefits of donating go beyond the economic rewards such as tax breaks and extend to include feelings of emotional satisfaction, enhanced self-esteem, recognition with the salient social network, and a felt increase in spiritual values (Arnett et al. 2003; Hibbert and Horne 1996). The general conclusion is that people donate in anticipation that the act of giving will make them feel better about themselves.

It is not surprising then that a perceived lack of reinforcement of the desired state leads to post-purchase NWOM. Indeed, ample evidence suggests instances of dissatisfaction after the donation has led to donors engaging in negative WOM (Wetzer et al., 2007a). The feelings of regret or remorse post donation are not uncommon and seem to stem from the idea of giving too much to the charity (Bennett 2009b). It is likely that just as positive WOM will reinforce other marketing communications and increase the spread of donations within the social network or identity group, the negative WOM will hinder and impede donations.

\section{The BASS model and negative word of mouth}

The original Bass Model and the subsequent literature in that diffusion stream focused on social pressure that induced members in the population to adopt an introduced product or behavior. Initiators either made an initial purchase or adopted a behavior without impetus from social pressure. Social pressure could be in the sheer number of previous adopters or pressure from social influence such as word of mouth. 
Working within these assumptions, as adapted for donating behavior, the Bass Model expresses the product's cumulative diffusion or behavior.

(a) Over (time) $\mathrm{m}$ initial people are donating for the first time. Donating behavior is infrequently occurring though it can be recurring.

(b) The likelihood of any initial donating behavior at $\mathrm{T}$ is represented by the function:

$[f(T)] /[l-F(T)]=P(T)=p+q / m Y(T)=p+q F(T)$.

Furthermore, as has been explicated in several articles (Bass 1969; Mahajan et al. 1990), the initial model then develops for discrete-time periods into for $t=1,2,3 \ldots$ Into:

$F(t)=\frac{1-e^{-(p+q) t}}{1+\frac{q}{p} e^{-(p+q) t}}$

The number of donors can be estimated by the above with the following behavioral assumptions:

(a) Initial donations can be by both "innovators" and "imitators."

(b) Initial donations of "innovators" are not influenced by the number of people who have already donated. They are "immune" to social influence. Donations by "imitators" are influenced by the number of previous donors.

(c) The coefficient of innovation is designated p, and q is the coefficient of imitation.

Attempting to incorporate NWOM into this model is not possible without changing some assumptions. One suggestion is to look at the effect of negative word of mouth as vaccination that reduces the potential population. While "innovators" are not influenced by the number of people who have already donated, the number of "innovators" and "imitators" would downwardly compress as the overall population potential reduces through an 'immunity' produced by negative word of mouth. Another suggestion is to view ' $q$ ' as partitioned with a positive and negative value, thus creating a summated ' $q$.'

These suggestions for incorporating negative word of mouth in the Bass model seem to have shortcomings when used to estimate parameters. The effect of modeling NWOM as a force, decreasing the size of the potential pool of donors, would lead to higher estimates of diffusion rate. This outcome occurs as the actual adoptions will be compared to this new lower overall potential population. Looking at lower rates of imitation from suggesting that the NWOM directly dampens the rate of imitation also has an issue. This assumption implies an additive function between NWOM rates of diffusion and positive word-of-mouth (PWOM) diffusion rates. The additive approach is not appropriate, as an imitator's choice of not adopting donating cannot be differentially ascribed only to the influence of negative word of mouth. For instance, an individual that hears of a positive review of a new charity from an acquaintance may or may not choose to donate to that charity. The lack of giving behavior is indistinguishable from the person that hears NWOM and subsequently does not gift.

\section{Adapting modern epidemiology models}

Building on recent approaches in modeling the spread of infectious disease may provide insights to WOM models. An adapted model might illuminate the simultaneous occurrence of PWOM and NWOM during the diffusion process. The $S I$ $R$ (susceptible, infectious, recovered) models of epidemiology look at the population as subdivided into compartments. Studying the rate of changes in the portion of the population in each compartment over time helps describe and analyze the spread of an infectious disease (Feng et al. 2011). These models seem particularly useful to marketing as they allow for the simultaneous inclusion of positive word of mouth and negative word of mouth in a population. One important note is that, as with the original diffusion models, these epidemiology models' marketing application requires a shift in viewpoint. Positive word of mouth is analogous to transmitting the disease or infection. In a marketing context, such an event (PWOM) is a positive marketing outcome. Furthering the analogy, the spread of negative word of mouth (NWOM) is comparable to immunization, preventing disease spread. NWOM is an undesirable event in marketing.

The earliest models of the spread of diseases had deterministic outcomes. So too for modern epidemiology models, which are also deterministic. These recent models are still based on the Kermack-McKendrick model or the core S I R Model in their simplest form. The distinguishing characteristic of these types of infectious disease models is computations of the theoretical number of people from a closed population moving through each compartment over time. The label of this class of models derives from the labels assigned to each compartment labels. In this most basic model, there are susceptible people $S(t)$, the number of people infected $I(t)$, and the number of people who have recovered $R(t)$. Hence, the S I R designation.

For this research paper, some notation adjustments are made to these labels in an overt attempt to shift viewpoints to the marketing context. As noted above, the desirability of the compartments shifts in a marketing context. The suggested shift in labeling helps emphasized this change in perspective. 
When considering the diffusion of a new product or service, marketing conceptualizes the target segment as the potential population instead of the susceptible population. The letter $S$ in the S I R model will change to P for potential as the designation in this research. The spread of positive word of mouth while equivalent to the infection rate is a sought outcome in a marketing context. The goal for marketing is for the target members to become purchasers. These initial customers are analogous to a patient zero, the person that first incurred the disease. These initial customers, through positive word of mouth, influence other potentials to become customers.

In this paper's estimation and validation section, the target population is potential donors to a non-profit. Some of these potentials may begin donating. They represent the start of the word-of-mouth process. Their PWOM may lead others in the potential group to begin donating. In the S I R model, the second compartment is infected; in the marketing target population, adoption of the marketing behavior leads to the letter A for the adopters. The donor in the estimation and validation model influenced by PWOM are the adopters. Purchasing behavior may discontinue at any point in time. Lapsing from participation in the marketing outcomes is analogous to Recovery. In the estimation of this model, donors may lapse and no longer donate. The designation for lapsed donors is $\mathrm{L}$.

These new labels change the $S I R$ model to the $P A L$ model for the marketing context. The explication of the $P A L$ model utilizes these labels in the assumptions of the "classic epidemic model" (Hethcote 2000). Those assumptions as adapted to the marketing context include:

- The population is fixed over time.

- The only way to leave the potential group is to become a customer. The only way to leave the customer group is to stop purchasing and not restart.

- Demographics such as gender, age, social status, and ethnicity are assumed for simplicity not to affect the probability of becoming a customer.

- There is no inherent condition that prevents one from becoming a customer.

- The members within the same geographic zone intermingle homogeneously.

This paper will use the following notation:

$P(t)$ is the number of potential donors at time $t$

$A(t)$ is the number adopting donating behavior at time $t$

$L(t)$ is the number of lapsed donors at time $t$

Building on this set of assumptions and using $N$ as the population size leads to these differential equations.

$\frac{\mathrm{d} P}{\mathrm{~d} t}=-\beta P(t) A(t)$ $\frac{\mathrm{d} P}{\mathrm{~d} t}=-\beta P(t) \gamma A(t)$

$\frac{\mathrm{d} L}{\mathrm{~d} t}=k \gamma A(t)$

where $k$ is the lapse rate and with $k$ assumed greater or equal to zero, $\alpha$ the probability of becoming a donor, $\gamma$ the average number of people a donor comes in word of mouth contact within each period, $\beta$ is the average number becoming donors as a result of positive word of mouth in each (with $\beta$ greater than or equal to zero), and

$P(t)+A(t)+L(t)=N$

Generally, these compartment models assume that as time approaches $\infty$, the potential compartment will approach zero and that lapsed compartment will approach $N$. More complicated is how the quantity changes in the adopting donating behavior compartment. We start by looking at the integral of equation three as through 0 to $t$, as in:

$k \int_{0}^{t} A(p) \mathrm{d} p=L(t)$

A simple transformation of Eq. (4) yields

$L(t)=N-P(t)-A(t)$

And combining Eqs. (5) and (6) gives,

$\int_{0}^{t} A(p) \mathrm{d} p=L(t)=N-P(t)-A(t)$

As previously noted, as $t$ approaches $\infty$, then $P(t)$ goes to zero, and $A(t)$ goes to zero, then the integral in Eq. (5) goes to a finite number.

That leaves the rate of change in the compartment of those adopting donating behavior as never zero or negative. The epidemiological designation of the rate of change is $\mathrm{R}_{0}$, also known as the effective reproductive number. This variable is the average of new infections produced by one infected individual during their entire period of being infectious. In the Bass Model, this variable is designated $\alpha$ and is known as the rate of imitation. Regardless of the nomenclature, the variable represents the average number of new people influenced by a single person with the existing condition. In this paper's P A L model, this variable represents the average number of people influenced to begin donating by an existing donor.

This rate can also be calculated for the whole population by the BASS model. However, under existing usages of that model, the negative influences would be subsumed when calculating the population $\alpha$ and imitation rate. This 
condition of the calculation of the rate of imitation is limiting. The Bass Model provides either the rate of imitation as positive only or a rate with positive and negative influence occurring simultaneously. There is no method of partitioning the rate of negative word of mouth in $\alpha$, the rate of imitation, in the BASS model. In the compartment model approach, the rate of transmission with only positive influence is calculable. Conditions of diffusion when with both positive and negative influence are present is also calculable. Only negative word of mouth and its influence is also calculable in the compartment model approach.

Finally, the compartmental models of epidemiology have extensions to allow for the inclusion of treatments, vaccinations, or both. Building on the experience of H1NI and other recent pandemics, the articles incorporate the impact of vaccination and/or treatment on the transmission of the virus (Feng et al. 2011; Gani et al. 2005; Lipsitch et al. 2007). These extensions model the treatment as a reduction in $\mathrm{R}_{0}$. A similar reduction is anticipated in $\alpha$, the rate of imitation, as negative word of mouth serves as an impediment to the spread of donating behavior being adopted.

The compartment models, social identity context, and mechanisms motivating word of mouth behavior suggest a level of interconnectedness during diffusion. It is undoubtedly true that internet access has promoted specific types of communications that heighten reach, but nothing in the literature suggests that this precludes in-person communication exchanges. Additionally, some of the $S I R$ model's saliency to marketing develops out of the assumption of spatially proximate interpersonal exchanges.

Many modern epidemiology models incorporate proximity as inherent in the transmission of infections or disease. Generally, models see the aggregation of clusters or nodes with varying levels of population density influencing transmission. This use of spatial proximity has seen applications in marketing and the density of clusters (Garber et al. 2004). Many models look at transmission outcomes as discrete and contained in each cluster, which in turn can aggregate into a report of spread throughout the whole population. An analogous approach using physical proximity in a market context adds managerial value to incorporating the $S I R$ model into marketing.

\section{Hypotheses and outcomes}

\section{Data context}

The data are from a US non-profit organization that uses Postal Service mail to gather contributions. The dataset contains 10 years of donor activity, including the size, frequency, and donation date from over 89,478 households. The data arrangement is by zip code and includes all fifty states and the District of Columbia. Starting in 1963, the United States Postal Service (USPS) clustered United States household delivery locations by geography (USPS 2013). Zipcode clusters based on household proximity are used by realtors and the United States Census Bureau (USPS 2013). Recording of the patterns of donations is by household with dates and dollar amounts provided for all households within the geographic proximity based on the zip code cluster.

The data record contains the date and the amount of the initial donation and amounts and dates of subsequent donations. Entries on donations include 20,039 of the roughly 42,000 post-office assigned five-digit zip codes (Center for International Earth Science Information Network 1996). Some calculations of average donation, number of donations, and other total data set calculations used the complete data set to estimate these parameters. Additionally, this study uses a random sample of 158 of these five-digit zip codes to estimate negative word of mouth calculations. The demographics of the random sample of households and population were compared to the entire data set, and no significant differences were found. Households are clustered within these zip codes. Analysis used geographic proximity by zip code. Geographic proximity positively indicates interpersonal word of mouth communications, as found previously in the marketing literature (Garber et al. 2004).

\section{Hypotheses and results}

The examination of the initial donations indicates a bimodal distribution. The most common donation pattern involves a small initial donation with subsequent donations at either that same level as the initial donation or slightly increasing over time. The other pattern of donations shows a sizeable initial gift. Pattern analysis reveals that large initial gifts are often one-time events without subsequent donations.

From the literature on buyer remorse and donating behavior, some individuals give more and then regret the donation amount (Bennett 2006, 2009a). There is no option for a purchase return, or the individual is reluctant to ask for a refund. Inspection shows that there are many sizeable initial donations in the population. Pattern analysis also reveals that any subsequent donations by that household do not follow these large donations.

We suggest that the difference in the size and type of donation indicates two types of adopters. The literature on donating behavior suggests motivation over the type of word of mouth may develop from each donor's assessment of their feelings after the initial donation. The larger the initial donation, research suggests, the higher the likelihood of dissatisfaction and then subsequent negative word of mouth. This pattern analysis and significant difference test provide 
evidence of a bimodal distribution of donors, spreading positive word of mouth and spreading negative word of mouth.

H1 Large initial donations that are not followed by any subsequent donations will have an average donation amount significantly larger than the population average donation.

The average household donation from those giving only one time (excluding the last period) is $\$ 38.25$, with a standard deviation of $\$ 6.85$. The overall population mean, including large first-time donations, have an average initial donation amount of $\$ 8.49$ with a standard deviation of $\$ 6.45$. Testing the difference in these two means indicates a statistically significant difference.

Beyond the actual initial donation behavior, we suggest an additional indicator of NWOM and PWOM. Over time, residents within close geographic proximity (same zip code) communicate with their neighbors about their charitable donations. The positive or negative nature of these communications will influence the spread of donations within that geographic area. We believe this pattern of donations within each zip code represents an additional indicator of the presence of NWOM or PWOM.

There are two types of word of mouth influencing donation behaviors. This paper suggests that small initial gifts lead to positive or possibly neutral feelings. Interpersonal communications in this group advance or neutrally impact imitation of the donating behavior. Clusters of households within this group will show a pattern of an increasing number of donors. The alternative suggestion is that large initial donations likely engender some remorse. Post-donating assessments likely lead to coping mechanisms such as discounting the charity during interpersonal communications. Clusters of households where a sizeable initial gift occurred will show a pattern of low or no spread of additional donors.

H2 The number of donors in zip codes with low initial donating will be significantly larger than the number of donors with a large initial donation.

The test of this hypothesis also indicates a difference between large initial donors and other donors. In zip codes with large initial donors, the average number of all donors over the 10 years is 11.8 per zip code, while the average number of donors in the other zip codes is 23.6. The test statistic for the difference is significant, $t=7.5, p<0.05$. This result suggests that the number of subsequent donations from all households in a zip code that had a large initial donation in its zip code is smaller than the subsequent donations in other zip codes.

The evidence from the two tests' outcomes indicates both negative and positive word of mouth as present in the donating patterns in this data. If both types of word of mouth are occurring in the overall population, the actual measure of influence, $\alpha$, the rate of imitation, would be affected by these different patterns of word of mouth communications. The rate of imitation of the population is likely downwardly compressed by the NWOM.

Categorization of the sample uses the criteria established above around the pattern of large initial donations (those with more than two standard deviations above the population mean as likely expressing NWOM). This coding approach dichotomizes the sample as zip codes likely to experience NWOM or PWOM. This approach will help further establish that both types of word of mouth are occurring as the presence of negative word of mouth and its subsequent suppression of imitation is not calculable by the BASS model. The zip codes with PWOM should not have the damping effect of NWOM. Subsequently, the rate of imitation in these zip codes should be higher than the population rate of imitation, which is a combination of PWOM and NWOM.

H3 The $\alpha$, the rate of imitation, for the population will be statistically significantly lower than $\alpha$, the rate of imitation for sample zip codes with positive word of mouth.

The hypothesis is fully supported. The $\alpha$, the rate of imitation for the population, is 0.08716 , as calculated using the Bass Forecasting model (De Bruyn 2020). Using the same forecasting approach $\alpha$, the rate of imitation for the zip codes coded as PWOM is 0.2847 with the s.d. of 0.155 . The test statistic is -6.41 with $p<0.05$. The zip codes categorized as likely having only PWOM have a significantly higher rate of imitation.

\section{Using the epidemiology model P A L}

These outcomes from above indicate the likely dual occurrence of NWOM and PWOM after the initial donation. The compartmental approach from recent epidemiology models builds on these outcomes - the family of compartmental models with versions incorporating treatments such as vaccinations or inoculations. These versions include equations allowing for calculating the number of treatments necessary to reduce $\mathrm{R}_{0}$ or the effective reproductive number. The equations incorporating treatments use $\mathrm{R}_{c}$, which is known as the control reproduction number (Feng et al. 2011).

A comparison between $R_{0}$ and $R_{c}$ helps policymakers determine the levels of control needed to prevent the disease's spread. Controls can be vaccinations, treatments, or a combination of both. In the marketing context, the issue is determining the level of inhibitors to the spread of diffusion. In this paper, the negative word of mouth is seen as a powerful restriction on diffusion in a population of donors after the initiation of the fund-raising campaign. 
Looking at the diffusion process in this marketing context in the compartment models of epidemiology helps develop the equations to determine negative word of mouth. Using an equation for calculating diffusion from the epidemiology literature (Feng et al. 2011) and changing the notation to match the P A L model of this paper is a start. The modification and relabeling help provide an equation for estimating the imitation rate when some form of an inhibitor (NWOM in the marketing context or vaccination in a disease context) is simultaneously present during diffusion in the population. The equation, as presented below, can, under certain conditions, provide estimates of NWOM.

$\alpha_{\mathrm{C}}=\beta_{0}(1-\eta \hat{p})\left(1-f_{0}\right) / \gamma_{\mathrm{u}}+\sigma \beta_{0}(1-\eta \hat{p}) f_{0} / \gamma_{\mathrm{tr}}$

where $\alpha_{\mathrm{C}}$ is the designation for the rate of imitation when an inhibitor to diffusion is present, $\beta_{0}$ is the conversion rate to donating behavior from word of mouth, $\gamma_{\mathrm{u}}$ lapse rate for donors not in contact with NWOM held constant as $1, \gamma_{\text {tr }}$ lapse rate for donors in contact with NWOM, held constant as $1, f_{0}$ portion of donors treated, held as zero as NWOM is vaccination, not a treatment, $\hat{p}$ proportion of the potentials inoculated against donating by NWOM, $\eta$ efficacy of the WOM, $\sigma$ Reduction in transmission due to NWOM, $p_{0}$ proportion of potentials inoculated by NWOM against donating behavior $=\eta \hat{p}$.

When an inhibitor or control, such as NWOM, is not present, then $f_{0}$ and $p_{0}$ are not occurring. This condition is equivalent to the untreated spread of disease. It should be evident that $\alpha_{\mathrm{C}}=\beta_{0}$ when the lapse rate is also not influenced by NWOM. From this conclusion, as in epidemiology, this equation creates a deterministic model. These models determine the efficacy of inoculations and/or the number needing vaccination to impede the spread of infectious disease. This paper presents a form of this equation to determine the level of NWOM present in the data.

Assuming that there is no form of 'treatment' as an intervention to change donating behavior to a lapse in donating, then $\mathrm{f}_{0}$ becomes zero. The equation then becomes:

$\alpha_{\mathrm{C}}=\beta_{0}(1-\eta \hat{p})+\sigma \beta_{0}(1-\eta \hat{p})$

Earlier, this paper found evidence that suggests that NWOM does control the diffusion of donating behavior. The sample provides an estimate of positive word of mouth, $\beta_{0}=0.2847$, on influencing donating behavior when a control such as NWOM is not present. As a further modification of equation eight, that specific estimate, $\beta_{0}=0.2847$, substitutes into the equation for beta. The right side of equation eight now accounts for the population as it simultaneously experiences NWOM and PWOM. The estimate of diffusion for the population with control present is $\alpha_{\mathrm{C}}=0.08716$. From the designations of equation notations from above where $p_{0}=\eta \hat{p}$, then equation eight above becomes: $.08716=.2847(1-\eta \hat{p})+\sigma .2847(1-\eta \hat{p})$

Since we know, $\alpha_{\mathrm{C}}<\beta_{0}$, the next step is to solve for combinations $\eta, \hat{p}$, and $\sigma$ that provides deterministic solutions in order for the equation hold. As a reminder, $\hat{p}$ is the proportion of the potentials inoculated against donating by NWOM and must be greater than or equal to zero, and $\eta$ efficacy of the WOM is positive. These assumptions mean that $\sigma$ must assume a negative value as Eq. 9 with simplification becomes:

$[.306147 /(1-\eta \hat{p}]-1=\sigma$

This transformation is dividing both sides of the equation by 0.2847 and $(1-\eta \hat{p})$.

The results of those solutions to equation nine are presented in Table 1 and shown in Fig. 1. This range of solutions suggests the exact level of influence for NWOM for combinations of $\eta, \hat{p}$.

The combinations of $\eta, \hat{p}$ with outcomes below the curve represent combinations of efficacy or proportion influenced. These show the underestimate of the impact of negative word of mouth. For example, with the estimate of $\eta$ and $\hat{p}$ change downward to 0.4 , the negative influence of word of mouth is lower, and the overall population estimate would rise to 0.093 , which is higher than the population estimate of $\alpha \mathrm{C}=0.08716$. Reversely, the combinations of $\eta, \hat{p}$ with results above the curve over-estimate the influence of negative word of mouth. There is an underestimate of the overall population diffusion of the adoption of donating behavior.

\section{Conclusions and implication}

This study represents one of the first large-scale studies to examine negative word of mouth and estimate the influence of behavior. The analysis is on over 219,000 measures of donating behavior across the United States and represents data from over 89,000 households. The paper legacy in the marketing literature from epidemiology. The use of the compartmental approach to investigating diffusion and word of mouth helps clarify researching the simultaneous occurrence of positive and negative word of mouth. Understanding the scope of negative word of mouth on early donating behavior provides insights into mitigating actions that might enhance campaign yields.

The context of donating behavior in diffusion models may seem to limit the generalizability of the study. However, the size of the dataset does somewhat offset this concern. Not only was the data from a large number of households with a significant number of behavioral measures, but it also had a breadth of coverage with all of the United States population 
Fig. $1 \sigma$ or solved NWOM for combinations of $\eta$ and $\hat{p}$

\section{Solutions for NWOM}

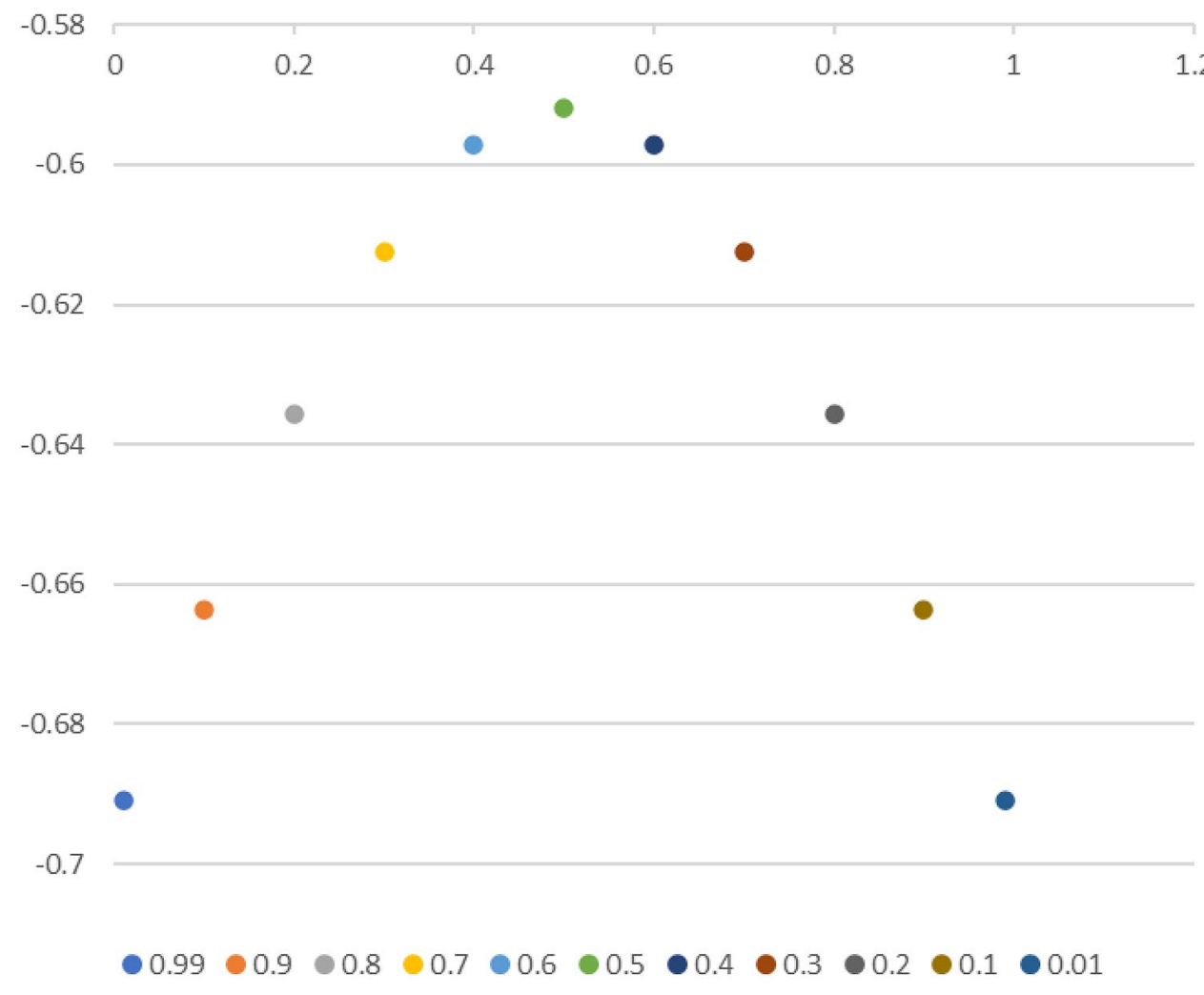

Table 1 Pairs of $\eta$ and $\hat{p}$ for solutions for $\sigma$ using Eq. 9

\begin{tabular}{lll}
\hline$\eta$ & $\hat{p}$ & $\sigma$ or solved NWOM \\
\hline 0.99 & 0.01 & -0.690793 \\
0.9 & 0.1 & -0.6635898 \\
0.8 & 0.2 & -0.63554 \\
0.7 & 0.3 & -0.612451 \\
0.6 & 0.4 & -0.59719 \\
0.5 & 0.5 & -0.5918 \\
0.4 & 0.6 & -0.59719 \\
0.3 & 0.7 & -0.612451 \\
0.2 & 0.8 & -0.63554 \\
0.1 & 0.9 & -0.6635898 \\
0.01 & 0.99 & -0.690793 \\
\hline
\end{tabular}

represented. The data are also longitudinal, covering donating behavior over 10 years.

The BASS diffusion model is limiting when attempting to estimate negative and positive word of mouth simultaneously. The influence of these two social pressures are not additive. The use of compartment models for epidemiology overcomes this limitation. The adaptation of $S I R$ to the marketing literature builds on the history of incorporating scientific models into marketing. The equations, and the models they represent, create a method of estimating NWOM in the marketing context.

Using that equation to estimate NWOM in this context demonstrates the significant influence of NWOM on donating behavior in this population's data. The range from -0.60 to -0.69 indicates just how powerful negative word of mouth can be. This strength is reflected in the lower diffusion rate in the overall population compared to diffusion in the sample with just positive word of mouth. It is not possible to estimate the actual losses in donating, but considering the overall amount of $\$ 1,830,000$ with an average of 20.52 per household over the 10 years, even a $10 \%$ increase could yield a substantial increase in donations.

Managers may also wish to look at understanding the reach of the broadcaster of the NWOM. The managers should realize that the more receivers of the message, the more likely the impact of that message: that increase in influence raises the necessity of intervention. Monitoring the efficacy of the NWOM message is also very relevant. Results show that even in low-reach conditions, an impactful message will have a high level of negative influence.

Managers should monitor the donating behavior, especially first-time donors. Approaching directly and personally, those initial donors giving amounts significantly beyond the 
average donation for an initial gift is a possible intervention. Establishing satisfaction and gaining an assurance that there is no remorse or regret of the amount of the donation can reduce the motivation behind NWOM. This additional effort can help mitigate the instigation of NWOM and its expanding impact on potential donors' population.

Beyond the realm of donating behavior, the use of compartment models may provide insights into diffusion. Many projections of diffusion rely on history and the diffusion patterns of similar products or services. However, once a product launch has become, the pattern of adoption may vary from projections.

Using the deterministic model based on the compartment model introduced could provide managers with insights. Looking at the patterns of adoption, and inserting possible influences of negative word of mouth, could produce a match in the adoption pattern. Should such a condition exist, the manager could feel confident in launching some primary research to discover if negative comments are occurring in the relevant social network.

\section{Limitations and future research}

The most immediate limitation is the reliance on inference about NWOM. The literature is clear that capturing expressions of negative word of mouth is a challenge. Future research could use an experimental design to confirm this paper's inference. Some evidence from the one paper that experimentally manipulated negative and positive word of mouth does tangentially support the assumption (Mahajan et al. 1984).

The dataset is geographically extensive, but in a unique setting in marketing. Future research needs to expand this type of analysis in other purchasing settings. The evidence for NWOM is indirect. A prospective study should establish a direct connection to negative word of mouth and purchase regret. Then, as in this data, the longitudinal occurrence and impact of this negative word of mouth must be traced.

The paper did calculate outcomes for $\eta$ and $\hat{p}$ separately across a broad range of options. Future research should attempt to determine more likely the levels of efficacy of the NWOM in various specific contexts. The actual number in the circle of the person spreading NWOM is another area of future research. The density of the population likely influences the reach of the broadcaster of NWOM.

Future research can also examine if the same issues occur in online environments. It may even be possible that tracing and monitoring NWOM and PWOM is facilitated by online data collection. There may be the ability to monitor the valence of word of mouth and then contact the instigator to establish the chosen messaging motivations.

\section{References}

Arndt, Johan. 1967. Role of Product-Related Conversations in the Diffusion of a New Product. Journal of Marketing Research 4 (3): 291-295.

Arnett, Dennis B., Steve D. German, and Shelby D. Hunt. 2003. The Identity Salience Model of Relationship Marketing Success: The Case of Nonprofit Marketing. Journal of Marketing 67 (2): 89-105.

Bass, Frank M. 1969. A New Product Growth Model for Consumer Durables. Management Science 15 (5): 216-227.

Bennett, Roger. 2006. Predicting the Lifetime Durations of Donors to Charities. Journal of Nonprofit \& Public Sector Marketing $15(1 / 2)$ : 45-67.

Bennett, Roger. 2009a. Regret and Satisfaction as Determinants of Lapsed Donor Recommencement Decisions. Journal of Nonprofit \& Public Sector Marketing 21: 347-366.

Bennett, Roger. 2009b. Regret and Satisfaction as Determinants of Lapsed Donor Recommencement Decisions. Journal of Nonprofit \& Public Sector Marketing 21 (4): 347-360.

Brauer, Fred, and Carlos Castillo-Chavez. 2013. Mathematical Models of Communicable Diseases. Philadephia: Society for Industrial and Applied Mathematics.

Brown, Jacqueline Johnson, and Peter H. Reingen. 1987. Social Ties and Word-of-Mouth Referral Behavior. Journal of Consumer Research 14 (3): 350-362.

Center for International Earth Science Information Network, Ciesin. 1996. Archive of Census Related Products (ACRP): 1990 ZIP Equivalency Files. Palisades, NY: NASA Socioeconomic Data and Applications Center (SEDAC).

Charlett, Don, Ron Garland, and Norman Marr. 1995. How Damaging is Negative Word of Mouth. Marketing Bulleting 6 (1): 42-50.

Chelminski, Piotr, and A. Coulter Robin. 2011. An Examination of Consumer Advocacy and Complaining Behavior in the Context of Service Failure. Journal of Services Marketing 25 (5): 361-370.

Chevalier, Judith A., and Dina Mayzlin. 2006. The Effect of Word of Mouth on Sales: Online Book Reviews. Journal of Marketing Research 43 (3): 345-354.

Bruyn, De., Gary L. Arnaud, and Arvind Rangaswamy Lilien. 2020. Enginius. Centre Hall, PA: Decision Pro Inc.

de Matos, Celso Augusto, and Carlos Alberto Vargas Rossi. 2008. Word-of-Mouth Communications in Marketing: A Meta-Analytic Review of the Antecedents and Moderators. Journal of Academy of Marketing Science 26 (4): 578-596.

Edison, Steven W., and Gary L. Geissler. 2011. An Investigation of Negative Word-Of-Mouth Communication Among Market Mavens. Journal of Behavioral Studies in Business 3 (April): $1-12$.

Feng, Zhilan, Sherry Towers, and Yang Yidng. 2011. Modeling the Effects of Vaccination and Treatment on Pandemic Influenza. The AAPS Journal 13 (3): 427-437.

Fibich, Gadi. 2016. Bass-SIR Model for Diffusion of New Products in Social Networks. Physical Review 94 (032035): 1-5.

Gani, Raymond, Helen Hughes, Douglas Fleming, Thomas Griffin, Jolyon Medlock, and Steve Leach. 2005. Potential Impact of Antiviral Drug Use During Influenza Pandemic. Emerging Infectious Diseases 11 (9): 1355-1362.

Garber, Tal, Jacob Goldenberg, Barak Libai, and Eitan Muller. 2004. From Density to Destiny: Using Spatial Dimension of Sales Data for Early Prediction of New Product Success. Marketing Science 23 (3): 419-428. 
Guy, Bonnie S., and Wesley E. Patton. 1989. The Marketing of Altruistic Causes: Understanding Why People Help. The Journal of Consumer Marketing 6 (1): 19-30.

Herr, Paul M., Frank R. Kardes, and John Kim. 1991. Effects of Wordof-Mouth and Product-Attribute Information on Persuasion. Journal of Consumer Research 17 (4): 454.

Hethcote, Herbert W. 2000. The Mathematics of Infectious Diseases. SIAM Review 42 (4): 599-653.

Hibbert, Sally, and Suzanne Horne. 1996. Giving to Charity: Questioning the Donor Decision Process. Journal of Consumer Marketing $13(2): 4-13$.

Kotler, Philip, and Sidney J. Levy. 1969. Broadening the Concept of Marketing. Journal of Marketing 33: 10-15.

Lipsitch, Marc, Ted Cohen, Megan Murray, and Bruce R. Levin. 2007. Antiviral Resistance and the Control of Pandemic Influenza. PLoS Medicine 4 (1): e15.

Liu, Yong. 2006. Word of Mouth for Movies: Its Dynamics and Impact on Box Office Revenue. Journal of Marketing 70 (3): 74-89.

Luo, Xueming. 2007. Consumer Negative Voice and Firm-Idiosyncratic Stock Returns. Journal of Marketing 71 (3): 75-88.

Mahajan, Vijay, Eitan Muller, and Frank M. Bass. 1990. New Product Diffusion Models in Marketing: A Review and Directions for Research. Journal of Marketing 54: 1-26.

Mahajan, Vijay, Eitan Muller, and Roger A. Kerin. 1984. Introduction Strategy for New Products with Positive and Negative Word-OfMouth. Management Science 30 (12): 1389-1404.

Pemberton, H. Earl. 1936. The Curve of Culture Diffusion Rate. American Sociological Review 1 (4): 547-556.

Richins, Marsha L. 1983. Negative Word-of-Mouth by Dissatisfied Consumers: A Pilot Study. Journal of Marketing 47 (1): 68-78.

Rogers, Everett M. 1962. Diffusion of Innovations. London: The Free Press.

Solis, Brian, and Alan Webber. 2012. The Rise of Digital Influence.

Stich, Lucas, Gerald Golla, and Alexandros Nanopoulos. 2014. Modelling the spread of negative word-of-mouth in online social networks. Journal of Decision Systems 23: 203-231.

Sundaram, D.S., Kaushik Mitra, and Cynthia Webster. 1998. Word-OfMouth Communications: A Motivational Analysis. Advances in Consumer Research 25: 527-531.
Tanny, S.M., and N.A. Derzko. 1988. Innovators and Imitators in Innovation Diffusion Modeling. Journal of Forecasting 7 (4): 225-234.

Taute, Harry, and Shaun McQuitty. 2004. Feeling Good! Doing Good! An Exploratory Look at the Impulsive Purchase of the Social Good. Journal of Marketing Theory and Practice 12 (2): 16-27.

USPS, Inspector General. 2013. "The Untold Story of the ZIP Code," 2021). https://permanent.fdlp.gov/gpo47009/rarc-wp-13-006.pdf.

Westbrook RA. 1987. Product/Consumption-Based Affective Responses and Postpurchase Processes. Journal of Marketing Research 24 (3): 258-270. https://doi.org/10.1177/0022243787 02400302.

Wetzer, Inge M., Marcel Zeelenber, and Rik Pieters. 2007a. "Never Eat In That Restraurant, I Did!": Exploring Why People Engage In Negative Word-Of-Mouth Communication. Psychology \& Marketing 24 (8): 661-680.

Wetzer, Inge M., Marcel Zeelenberg, and Rik Pieters. 2007b. "Never Eat In That Restraurant, I Did!” Exploring Why People Engage in Negative Word-Of-Mouth Communications. Psychology \& Marketing 24 (8): 661-680.

Publisher's Note Springer Nature remains neutral with regard to jurisdictional claims in published maps and institutional affiliations.

John Andy Wood, Ph.D joined James Madison University in July of 2014 as Professor of Marketing. He previously taught at West Virginia University and Georgia State University. He has been a visiting lecturer at the University of Otago in New Zealand, Antwerp University in Belgium, and the Royal University for Women in Bahrain. Before his academic career, he spent 17 years in the Building Materials sector, ending his career as the Director of Sales and Marketing for CSR. Andy's research is in Professional Sales with an emphasis on nonverbal signals that lead to trustworthiness. Much of this research focuses on the neuro-marketing and neurological aspects of facial displays. He has several papers on analytics and methods in marketing. His business experience and personal travel have led to several research studies and projects helping companies with international market selection and entry. 